Gómez García, M., Boumadan Hamed, M., Poyatos Dorado, C. \& Soto Varela, R. (2020). Formación docente en línea a distancia. Un análisis de los perfiles y la opinión de los profesores. Revista Electrónica Interuniversitaria de Formación del Profesorado, 23(2), 95-111.

DOI: https://doi.org/10.6018/reifop.423001

\title{
Formación docente en línea a distancia. Un análisis de los perfiles y la opinión de los profesores
}

\author{
Melchor Gómez García ${ }^{(1)}$, Moussa Boumadan Hamed ${ }^{(1)}$, César Poyatos Dorado ${ }^{(1)}$, Roberto \\ Soto Varela ${ }^{(2)}$ \\ ${ }^{(1)}$ Universidad Autónoma de Madrid, ${ }^{(2)}$ Universidad Alfonso X
}

\section{Resumen}

Los cursos de formación en línea son considerados en ocasiones como poco motivadores debido a la ausencia de relación con el tutor y entre los participantes del curso. Pero cada día hay más cursos a distancia para formación docente, por ello, el objetivo del presente estudio es conocer qué perfil de docentes opta por la formación en línea y qué opinión genera en ellos.

Se han analizado cincuenta cursos en línea pertenecientes a la oferta formativa de la Consejería de Educación de Madrid, obteniendo datos de los cuestionarios en línea realizados al final de cada uno de esos cursos.

Se ha encontrado que esta oferta formativa en línea está promovida y canalizada fundamentalmente desde la web y los servicios de la propia consejería y que lo que más se valora por parte de los profesores que se están formando son los contenidos y actividades, más que el formato del propio curso o las tecnologías en que se apoya. Los cursos con temáticas pedagógicas son los que más se valoran, por encima de los tecnológicos.

Las conclusiones apuntan hacia la necesidad de un refuerzo de la parte tecnopedagógica en los cursos que se desarrollan en entornos de aprendizaje en línea.

\section{Palabras clave}

Formación a distancia; formación de docentes en activo; enseñanza multimedia; formación continua. 


\section{Online teacher training. An analysis of teachers'profiles and teachers'opinion.}

\section{Abstract}

The lack of a relationship between the tutor and the course participants makes this type of environment unmotivating for most online training course participants. But every day there is an increasing offer of online courses for teacher training, so the aim of the study is to know the profile of teachers who opt for online training and their opinion about them.

Fifty online courses belonging to the educational offer of the Regional Ministry of Education of Madrid have been analysed, obtaining data from the online surveys carried out at the end of each of these courses.

It has been found that this online training offer is promoted and channelled mainly from the web and the services of the council itself. What is most valued by the teachers surveyed are the contents and activities, rather than the format of the course or the technologies on which it is based. The courses with pedagogical themes are the ones that are most valued above the technological ones.

The conclusions suggest that a boost in the technopedagogical part of the courses developed in online learning environments is needed.

\section{Key words}

e-learning; teacher training; multimedia education; lifelong learning.

\section{Introducción}

Los docentes son el colectivo que más número de horas de formación voluntaria realiza fuera de su horario laboral. Por esta razón es importante definir los perfiles de los docentes que se forman, y en especial aquellos que lo hacen fuera de su horario lectivo, para conocer la opinión que tienen respecto a la formación en línea, que es la más flexible en cuanto a horario y localización física.

Según la ley española, si la formación no es obligatoria para la empresa se podrá realizar fuera de la jornada laboral, y si lo es, deberá impartirse dentro del horario de trabajo. En los centros educativos de Secundaria la formación de los docentes es imprescindible, pero no obligatoria, por lo que se realiza mayoritariamente fuera del horario de trabajo y en formato a distancia.

Para ser docente se debe realizar una formación de Grado, y en muchos casos completar con un Posgrado en Educación. Tras esta formación inicial, el docente debe seguir con su formación durante toda su vida laboral para mantenerse actualizado y para cubrir determinados complementos salariales.

Este trabajo analiza la opinión de más de 7500 docentes en una potente investigación desde el punto de vista educativo al abarcar un gran segmento de la población analizada. Y también es una investigación socialmente interesante pues mejorará el ecosistema docente al delimitar qué elementos promueven que los profesores se formen y por tanto sean mejores profesionales. 


\section{Revisión teórica}

\section{Formación continua y Competencia Digital Docente}

Hace más de una década un estudio de McKinsey comparó a los considerados diez mejores sistemas educativos del mundo. Esta investigación destacó como principal conclusión que el nivel educativo de un país depende de la formación, motivación y aprendizaje permanente de sus profesores. Señala además, que los países con mejor calidad de enseñanza facilitan abundante formación práctica a los recién llegados a la carrera docente y fomentan la formación permanente para todos.

Desde el siglo pasado ya existen estudios y literatura abundante que marca a los profesores como la pieza clave del sistema. Fullan (1992) ya había ubicado al perfil docente como uno de los máximos responsables en la aspiración de colocar al aprendiz en el centro del proceso de aprendizaje. Hargraves (2005) reflexionó en profundidad sobre el papel que desempeña el docente en los planteamientos de una institución educativa que denominó "las escuelas que aprenden".

Con el nuevo siglo llegó el avance de la sociedad que ha ubicado a las Tecnologías de la Información y Comunicación (TIC) como un factor a tener en cuenta en cualquier sector, y especialmente en el de la educación (Castells, 2003). También Coll, Onrubia y Mauri (2007) destacaron en este sentido la generación de nuevos nichos de aprendizaje, nacidos de los espacios digitales en los que las nuevas generaciones invierten gran parte de su tiempo.

El Consejo de la Unión Europa que marca las políticas del continente ha planteado con el nuevo siglo un renovado enfoque de las habilidades de los ciudadanos europeos, y pone especial énfasis en el papel que las tecnologías digitales tienen para la formación permanente. Las 8 competencias elementales para el aprendizaje permanente son definidas como una combinación de conocimientos, capacidades y actitudes adecuadas al contexto, y entre estas habilidades está la competencia digital y competencia en tecnología. (Consejo de la UE, 2018, p. 7).

En el ámbito docente se hacen importantes aquellas capacidades o destrezas relacionadas con el uso de las herramientas tecnológicas en el aula, que son distintas en muchos aspectos del uso que se le puede dar a estas en un ambiente doméstico (Blau y Shamir-Inbal, 2017; Solís de Ovando y Jara, 2019). Según Cabero-Almenara, Estrada-Vidal y Gutiérrez-Castillo (2017), la competencia digital docente está vinculada con todas aquellas habilidades, actitudes y conocimientos requeridos por los profesionales de la docencia en un mundo digitalizado.

En este sentido surge el Marco Europeo de Competencia Digital del Profesorado «DigCompEdu» (Redecker y Punie, 2017). Un documento que tiene como finalidad principal apoyar a los estados miembros de la UE en sus líneas de trabajo vinculadas con promover la competencia digital docente e impulsar la innovación en educación. Sus áreas competenciales son compromiso profesional, recursos digitales, pedagogía digital, evaluación y retroalimentación, empoderar a los estudiantes, y facilitar la competencia digital de los estudiantes.

Por lo tanto para que los recursos tecnológicos apoyen la innovación pedagógica, los docentes deben tener una formación técnica en herramientas digitales y una formación didáctica que les dote del saber hacer pedagógico mediado por las TIC. (Del Prete, CaberoAlmenara y Halal, 2018; Rodríguez, Raso y Ruiz-Palmero, 2019). 


\section{Los nuevos escenarios de formación mediados por las TIC}

Diferentes trabajos e investigaciones actuales confirman el importante grado de aceptación que despiertan en los docentes los entornos digitales de aprendizaje y definen las diversas variables que determinan su uso y aceptación (Park, Nam y Cha, 2012; Alharbi y Drew, 2014; Persico, Manca y Pozzi, 2014; Tarhini, Hone, y Liu, 2014; Valencia, Benjumea, y Rodríguez, 2014; Kang y Shin, 2015). De entre esas variables destaca el grado de manejo de las herramientas que un espacio virtual presenta (Deniz y Algan, 2007), así como el dominio para un uso pedagógico y contextualizado (Celik y Yesilyurt, 2013). Los motivos son variados, pero se destacan la flexibilidad de horarios y el fácil acceso desde diferentes lugares, como los elementos más importantes para su buena acogida entre los docentes. Estas características se adaptan especialmente bien al modelo de formación continua del profesorado, que se realiza fuera del horario lectivo y generalmente lejos del centro escolar.

Estos entornos de aprendizaje digital son fundamentalmente plataformas que gestionan una secuencia formativa de e-learning. Permiten y favorecen cambios en las formas de entrega y comunicación (Park, 2014), desarrollan funcionalidades con herramientas que mejoran la experiencia de aprendizaje y facilitan que dicho aprendizaje pueda ser colaborativo (Sinclair y Aho, 2018). Pero el desarrollo de su uso didáctico sigue siendo limitado después de décadas (Rienties et al., 2014; Parsons, 2017).

Estas plataformas digitales para la formación docente suelen favorecer una dinámica de trabajo basada en la acción de los aprendices y la colaboración entre iguales (Ramos-Pardo, Sánchez y Sánchez, 2012), y facilitan plantear enfoques pedagógicos innovadores, que es lo que realmente permite ser eficientes en la formación.

\section{Contexto}

La formación docente en España se oferta en diferentes formatos: presencial, mixta o a distancia. Pero fundamentalmente es presencial. Por ello se considera una prioridad estratégica desarrollar cursos en línea que faciliten al profesorado en activo el acceso y seguimiento de los programas de formación, permitiendo una flexibilidad tanto geográfica como de horario.

Con este fin, la Consejería de Educación de la Comunidad de Madrid oferta anualmente diversos cursos de formación en línea a distancia a través de su plataforma digital. Entre 2017 y 2018 se ofrecieron una cincuentena de cursos tutorizados en modalidad online, además de los cursos presenciales y los cursos en línea masivos (MOOC), modalidades que no abordaremos en este artículo.

Cada curso sigue una estructura común diseñada desde el Centro Regional de Innovación y Formación (CRIF) y están asistidos por unos tutores que también han sido formados en el mismo Centro. Todas las actividades se realizan en línea y la comunicación se realiza también por Internet. Al finalizar el curso se ofrece un cuestionario para evaluar los diferentes aspectos del curso y las razones por las cuales se accedió al proceso formativo.

Los cursos en línea se ofrecen desde la plataforma digital docente de la Consejería de Educación y los participantes acceden a los distintos módulos del curso y a las herramientas de comunicación en un entorno único. Además, los cursos se acompañan de manera opcional con herramientas externas a la plataforma, como redes sociales o foros.

Metodología 


\section{Participantes}

Los participantes de este estudio fueron los profesores que tomaron parte en los cursos de formación en línea ofrecidos por la Consejería de Educación de la Comunidad de Madrid en los cursos 2017-18 y 2018-19. Por lo tanto, se trata de un muestreo no probabilístico.

Tabla 1.

Participantes en cursos en línea. Elaboración propia

\begin{tabular}{|c|c|c|c|c|}
\hline & & $\begin{array}{c}\mathrm{N} \\
\text { participan }\end{array}$ & $\begin{array}{c}\mathrm{N} \text { responden } \\
\text { encuesta }\end{array}$ & $\begin{array}{c}\text { \% responden } \\
\text { encuesta }\end{array}$ \\
\hline \multirow[t]{27}{*}{ Nombre curso } & Aprende a Buscar & 56 & 55 & $98,2 \%$ \\
\hline & Aprend. Basado Proyect & 844 & 811 & $96,1 \%$ \\
\hline & Aprend. Cooperativo & 131 & 129 & $98,4 \%$ \\
\hline & Aprend. Servicio & 177 & 135 & $76,27 \%$ \\
\hline & Arte Colaborativo Digital & 24 & 24 & $100 \%$ \\
\hline & Cedro & 157 & 157 & $100 \%$ \\
\hline & Convivencia & 472 & 472 & $100 \%$ \\
\hline & Curso Tutores & 245 & 245 & $100 \%$ \\
\hline & Del Papel a la Nube & 330 & 330 & $100 \%$ \\
\hline & Diseño Aprendizajes & 36 & 34 & $94,4 \%$ \\
\hline & Educa Madrid & 557 & 524 & $94,1 \%$ \\
\hline & Espacios Web & 148 & 131 & $88,5 \%$ \\
\hline & Evaluación & 95 & 87 & $91,5 \%$ \\
\hline & Evaluar para Aprender & 744 & 662 & $88,9 \%$ \\
\hline & Flipped Classroom & 1014 & 1013 & $99,9 \%$ \\
\hline & Función Tutorial & 102 & 66 & $64,7 \%$ \\
\hline & Funcionario Prácticas & 31 & 31 & $100 \%$ \\
\hline & Inst. Nacional Estadística & 113 & 105 & $92,9 \%$ \\
\hline & Moodle & 1208 & 1208 & $100 \%$ \\
\hline & Paisajes Aprendizaje & 652 & 588 & $90,1 \%$ \\
\hline & Propiedad Intelectual & 83 & 83 & $100 \%$ \\
\hline & Tecnol. Program. y Rob. & 46 & 42 & $91,3 \%$ \\
\hline & Transformación Digital & 43 & 43 & $100 \%$ \\
\hline & Web Aula & 190 & 174 & $91,5 \%$ \\
\hline & Web Centro & 92 & 90 & $97,8 \%$ \\
\hline & Web Docente & 294 & 274 & $93,2 \%$ \\
\hline & Total & 7810 & 7513 & $96,2 \%$ \\
\hline
\end{tabular}

Al final del proceso de capacitación, desde la propia plataforma formativa se plantea el cuestionario del estudio a los profesores que completaron el curso. El número total de participantes fue de 7810 participantes, de los que respondieron el cuestionario 7513 . Esta alta participación es debida a que el cuestionario está insertado en el mismo curso, y es requisito para poder finalizar con éxito la experiencia formativa. 


\section{Instrumento}

Los datos fueron recolectados a través de un cuestionario en línea ubicado en el último módulo de cada curso. Dicho cuestionario fue diseñado por un grupo de discusión formado por profesionales del Centro Regional de Innovación y Formación, que se reunió durante varias sesiones. En la primera se definió el tipo de cuestionario (áreas de interés, número de elementos, estilo de escritura, escala de calificación y formato de presentación). El cuestionario se pasó a un grupo piloto de personas que habían realizado alguno de los cursos en línea. En la siguiente sesión se redactaron los diferentes elementos y su disposición en el cuestionario. Se incluyeron diferentes tipos de preguntas, como la opción múltiple, preguntas cerradas y abiertas. Las preguntas de valoración del curso se realizaron con una escala Likert de 6 puntos. El producto resultante fue sometido a una consulta de expertos externos (método Delphi), que completó la construcción de la herramienta, logrando así un alto grado de validez para el propósito del cuestionario.

Una vez validado, el cuestionario se digitalizó a través de los formularios de Google Drive. En dicho cuestionario se recopilaron datos de los participantes sobre su percepción del curso, su procedencia, la información para realizarlo y si recomendarían este tipo de formación a otros compañeros o no.

La fiabilidad del cuestionario se analizó utilizando el coeficiente Alfa de Cronbach. El marcador fue de 0.961, lo que permitió establecer la consistencia interna del instrumento.

\section{Trabajo de campo}

Durante los cursos lectivos 2017-18 y 2018-19 se han llevado a cabo 50 acciones formativas a distancia con soporte digital en la Consejería de Educación de la Comunidad de Madrid. En cada curso se ha realizado un cuestionario con una parte específica y una parte común. Todos los cuestionarios realizados en estos dos años se han almacenado en una base de datos, que se ha analizado posteriormente por el equipo investigador.

\section{Resultados}

Los cursos en línea para formación de profesores que ofrece el Centro Regional de Innovación y Formación tienen un margen de flexibilidad en cuanto al número de participantes. Aquellos cursos con mayor demanda son más repetidos en el tiempo o ven ampliada su ocupación a través de la asignación de más tutores.

Tras clasificar los cursos de la oferta formativa en 4 grupos según la temática de cada curso (tecnológicos, metodológicos, pedagógicos y específicos), se comprueba que el número de inscritos es diferente en función de dicha temática. Se observa una clara preferencia de cursos de temática Metodológica y temática Tecnológica, con cerca de un 40\% para cada uno de las temáticas.

Las temáticas menos demandadas han sido las de contenidos específicos de área de conocimiento, que apenas han supuesto el $5 \%$ del total.

Los docentes son un colectivo que tienen inquietud por su formación, y a la hora de formarse es importante el canal que les lleva al curso concreto que realizan. Analizando las distintas fuentes de información, se comprueba que el canal de comunicación que tiene mayor influencia es la web institucional del propio Centro Regional de Innovación y Formación (CRIF), que orienta a casi la mitad de los participantes.

Tabla 2.

Participantes en cursos según temática. Elaboración propia. 


\begin{tabular}{llrr}
\hline & Frecuencia & Porcentaje \\
\hline Temática & Específicos & 376 & 5,0 \\
& Metodológico & 3022 & 40,2 \\
Pedagógico & 1287 & 17,1 \\
Tecnológico & 2828 & 37,6 \\
Total & 7513 & 100,0 \\
\hline
\end{tabular}
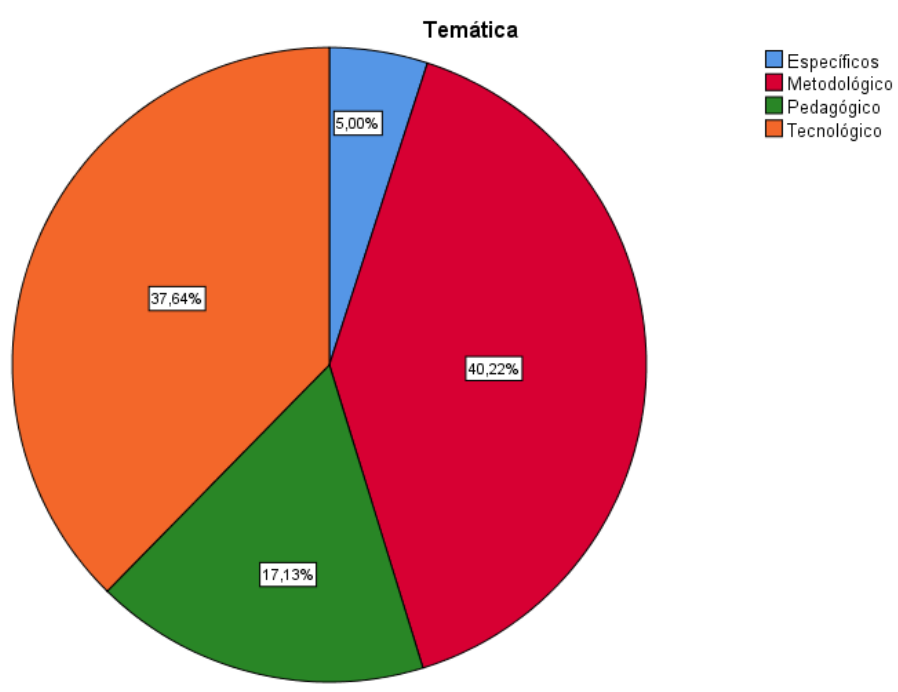

Figura 1. Participantes en cursos por temáticas

Tabla 3.

Conocimiento del curso

\begin{tabular}{llrr}
\hline & & N & \multicolumn{1}{c}{$\%$} \\
\hline Tuve conocimiento de & Claustro / Centro & 1071 & $14,3 \%$ \\
este curso a través de: & Compañeros & 281 & $3,7 \%$ \\
& Correo de EducaMadrid & 1153 & $15,3 \%$ \\
& Múltiples vías & 929 & $12,4 \%$ \\
& NC & 13 & $0,2 \%$ \\
& Otras páginas web & 427 & $5,7 \%$ \\
& Página web del CRIF & 3583 & $47,7 \%$ \\
& Redes Sociales & 56 & $0,7 \%$ \\
& Total & 7513 & $100,0 \%$ \\
\hline
\end{tabular}

El claustro de profesores y los correos que envía la consejería son los otros dos elementos destacados en la captación de formación para los docentes, con cerca del 15\% para cada uno. Las redes sociales, tan mediáticas y extendidas, apenas tienen influencia a la hora de informar a los docentes en temas de formación. 


\section{Tuve conocimiento de este curso a través de:}

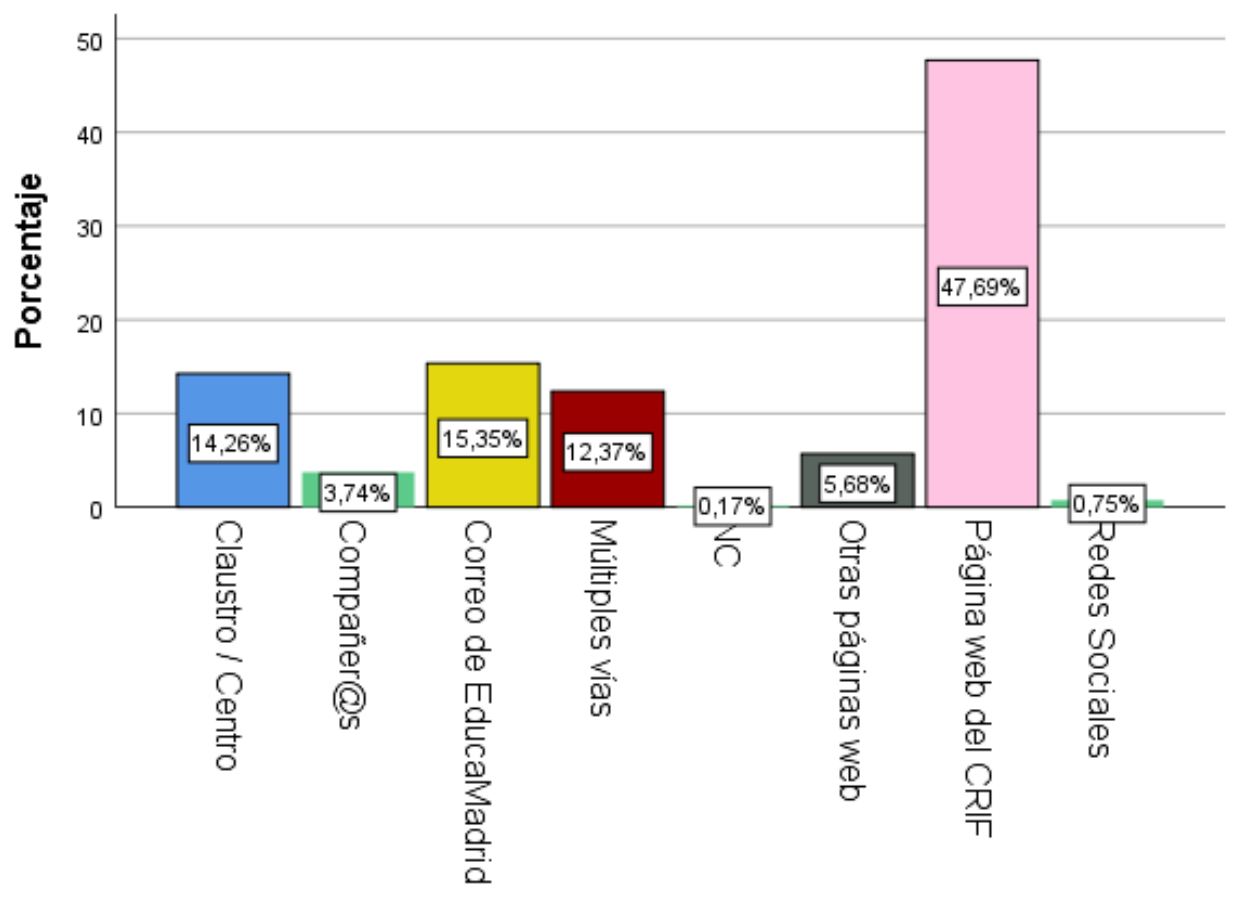

Figura 2. Diagrama de medios de acceso al curso

Un elemento importante para calibrar la eficacia de la formación recibida es la transferencia al aula de lo aprendido en el curso. A la hora de poner en práctica lo aprendido los resultados nos destacan al curso de temática tecnológica "Del papel a la nube", muy por encima del resto de la oferta formativa.

De lejos le siguen "Aprender a Buscar" - curso también tecnológico- y "Aprendizaje cooperativo" -curso de temática metodológica-.

Tabla 4.

Puesta en práctica del curso

\begin{tabular}{|c|c|c|c|c|c|}
\hline & & & $\begin{array}{l}\text { Aún no he } \\
\text { tenido } \\
\text { oportunidad, } \\
\text { pero voy a } \\
\text { hacerlo } \\
\%\end{array}$ & $\begin{array}{l}\text { No } \\
\%\end{array}$ & $\begin{array}{l}\text { Sí } \\
\%\end{array}$ \\
\hline & & $\%$ & $\%$ & $\%$ & $\%$ \\
\hline \multirow[t]{7}{*}{ Nombre curso } & Aprende a Buscar & $0,0 \%$ & $49,1 \%$ & $1,8 \%$ & $49,1 \%$ \\
\hline & $\begin{array}{l}\text { Aprendizaje Basado } \\
\text { Proyectos }\end{array}$ & $0,1 \%$ & $75,8 \%$ & $7,5 \%$ & $16,5 \%$ \\
\hline & Aprendizaje Cooperativo & $0,0 \%$ & $48,8 \%$ & $1,6 \%$ & $49,6 \%$ \\
\hline & Aprendizaje Servicio & $0,0 \%$ & $66,7 \%$ & $8,1 \%$ & $25,2 \%$ \\
\hline & Arte Colaborativo Digital & $0,0 \%$ & $12,5 \%$ & $0,0 \%$ & $87,5 \%$ \\
\hline & Cedro & $0,0 \%$ & $56,1 \%$ & $7,6 \%$ & $36,3 \%$ \\
\hline & Convivencia & $0,0 \%$ & $48,7 \%$ & $7,8 \%$ & $43,4 \%$ \\
\hline
\end{tabular}




\begin{tabular}{lllll} 
Curso Tutores & $0,0 \%$ & $61,2 \%$ & $11,0 \%$ & $27,8 \%$ \\
Del Papel a la Nube & $0,0 \%$ & $24,8 \%$ & $3,6 \%$ & $71,5 \%$ \\
Diseño Aprendizajes & $0,0 \%$ & $70,6 \%$ & $17,6 \%$ & $11,8 \%$ \\
Educa Madrid & $0,2 \%$ & $70,0 \%$ & $9,9 \%$ & $19,8 \%$ \\
Espacios Web & $0,0 \%$ & $63,4 \%$ & $8,4 \%$ & $28,2 \%$ \\
Evaluación & $0,0 \%$ & $66,7 \%$ & $3,4 \%$ & $29,9 \%$ \\
Evaluar para Aprender & $0,2 \%$ & $54,8 \%$ & $2,6 \%$ & $42,4 \%$ \\
Flipped Classroom & $0,1 \%$ & $59,7 \%$ & $2,8 \%$ & $37,4 \%$ \\
Función Tutorial & $0,0 \%$ & $51,5 \%$ & $13,6 \%$ & $34,8 \%$ \\
Funcionario Prácticas & $0,0 \%$ & $90,3 \%$ & $3,2 \%$ & $6,5 \%$ \\
Instituto Nacional & $0,0 \%$ & $85,7 \%$ & $4,8 \%$ & $9,5 \%$ \\
Estadística & & & & \\
Moodle & $0,5 \%$ & $72,4 \%$ & $5,0 \%$ & $22,1 \%$ \\
Paisajes Aprendizaje & $0,2 \%$ & $74,0 \%$ & $4,4 \%$ & $21,4 \%$ \\
Propiedad Intelectual & $0,0 \%$ & $59,0 \%$ & $8,4 \%$ & $32,5 \%$ \\
Tecnología Programación & $0,0 \%$ & $59,5 \%$ & $26,2 \%$ & $14,3 \%$ \\
y Robótica & & & & \\
Transformación Digital & $0,0 \%$ & $67,4 \%$ & $20,9 \%$ & $11,6 \%$ \\
Web Aula & $0,0 \%$ & $75,3 \%$ & $4,0 \%$ & $20,7 \%$ \\
Web Centro & $0,0 \%$ & $43,3 \%$ & $37,8 \%$ & $18,9 \%$ \\
Web Docente & $0,0 \%$ & $75,5 \%$ & $3,6 \%$ & $20,8 \%$ \\
Total & $0,1 \%$ & $63,7 \%$ & $6,1 \%$ & $30,0 \%$ \\
\hline
\end{tabular}

De manera global, podemos decir que el 30\% de los docentes ha transferido al aula lo aprendido en los cursos. En estos resultados es importante la temática del mismo, y lo podemos comprobar en la tabla 5 , que muestra este mismo análisis de transferencia pero desde un punto de vista agrupado. Son claramente los cursos con contenidos pedagógicos los más efectivos a la hora de ponerlos en práctica en el aula, superando en casi 15 puntos porcentuales al resto de categorías.

Tabla 5.

Puesta en práctica del curso por temáticas.

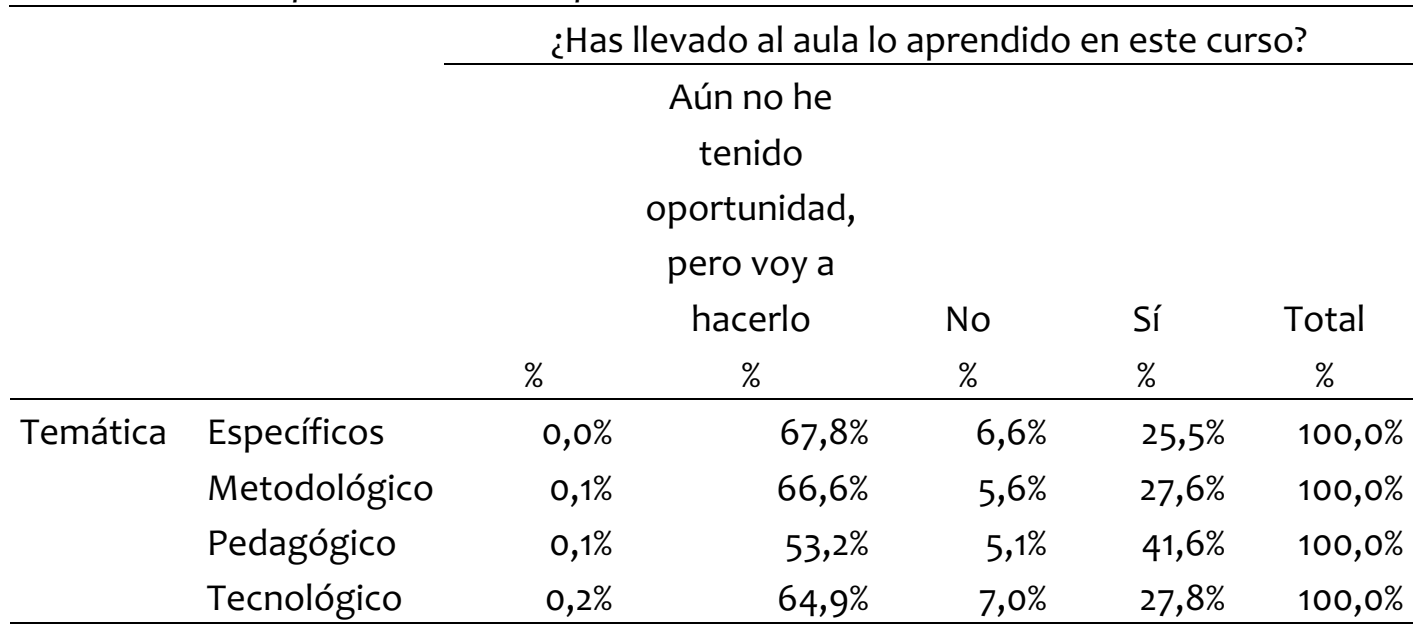

Si observamos la valoración que se ha hecho de diferentes parámetros de los cursos veremos una satisfacción alta y cercana al 5 (sobre 6) en casi todos los aspectos valorados y en prácticamente la totalidad de los cursos realizados. 
Tabla 6.

Valoración Global por Curso

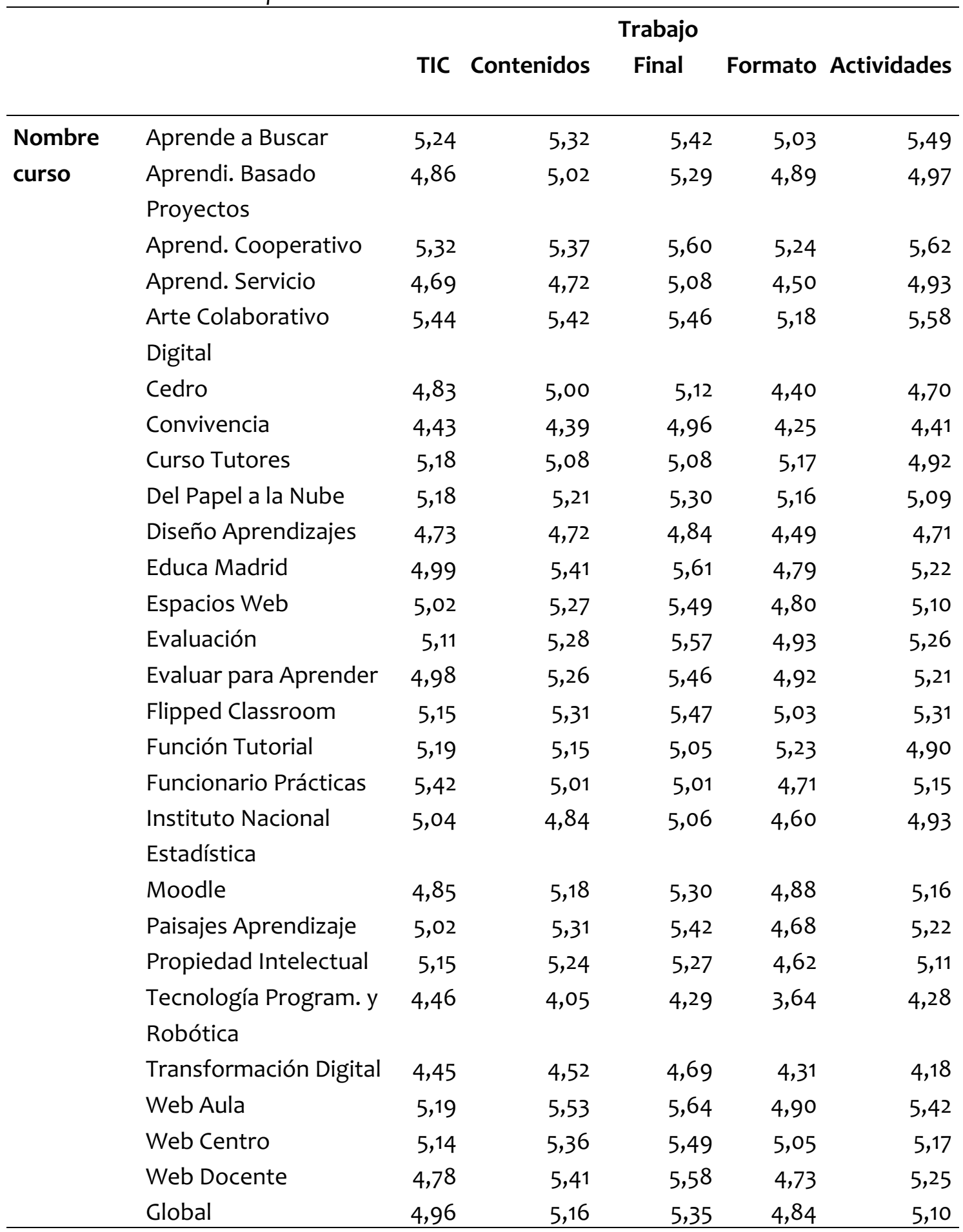

Si nos fijamos en la valoración de los distintos aspectos de los cursos en su conjunto observamos de manera más clara que las TIC y el formato del curso son los elementos menos valorados, y el diseño del trabajo final el mejor valorado. 
En la figura 3 se puede observar que las medias y las medianas de estos cinco aspectos, tienen valores altos, y una alta concentración de las medidas, destacando los parámetros de los contenidos, las actividades y el trabajo final.

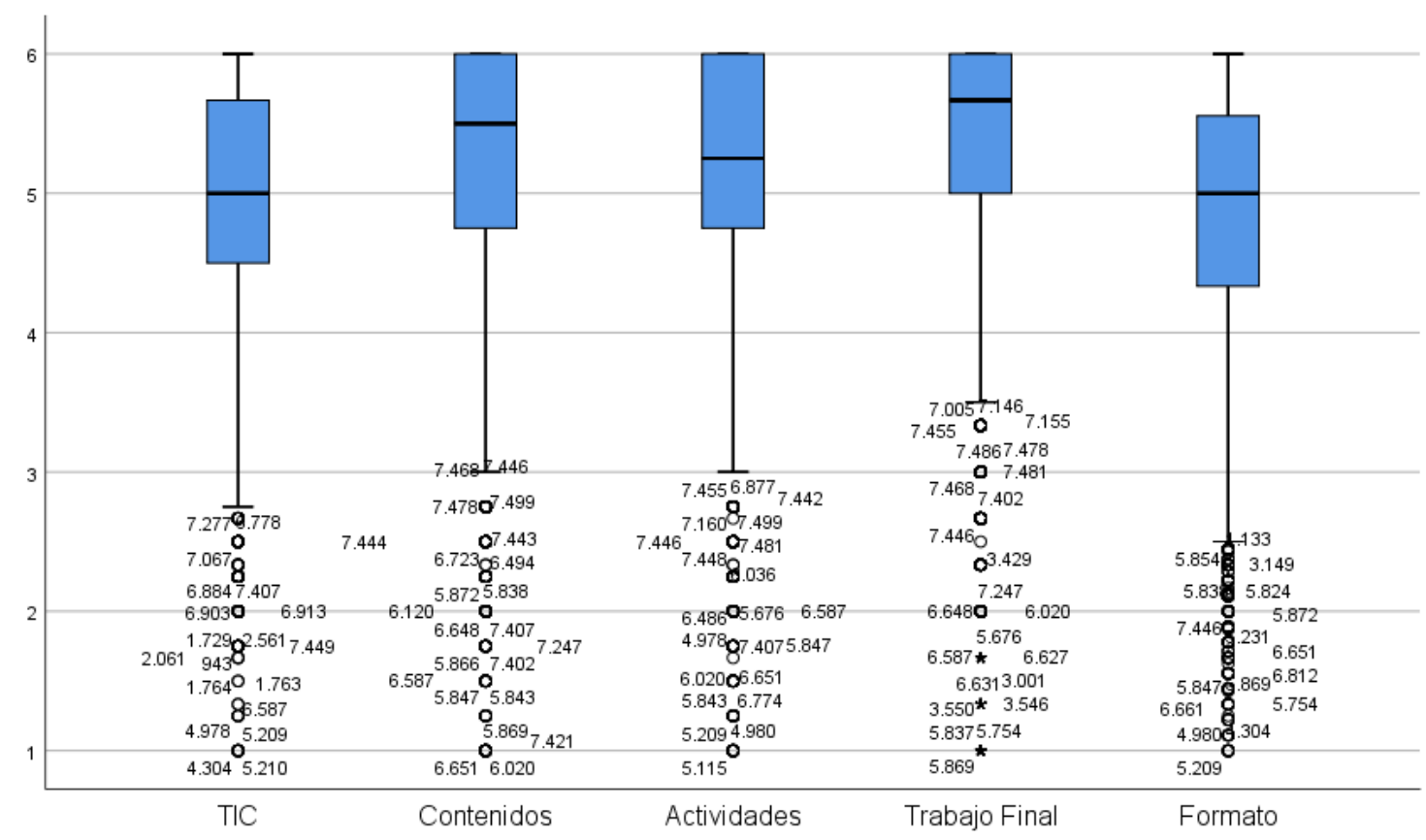

Figura 3. Comparación de las valoraciones en los cursos.

Finalmente nos fijamos en la satisfacción del profesor con el curso realizado, a través de la convicción del propio participante en el curso para recomendarlo a otros docentes. Este análisis se realizó en una escala de 10, por la familiaridad que los docentes tienen con este formato decimal para evaluar.

Tabla 7.

Recomendaría el curso

\begin{tabular}{llr}
\hline & & $\begin{array}{c}\text { ¿Recomendarías este } \\
\text { curso a otro docente? } \\
\text { Media }\end{array}$ \\
\hline Nombre curso & \multicolumn{2}{c}{8,69} \\
\cline { 2 - 3 } & Aprende a Buscar & 7,63 \\
Aprend. Basado Proyectos & 8,81 \\
Aprend. Cooperativo & 7,74 \\
Aprend. Servicio & 9 \\
Arte Colaborativo Digital & 7,89 \\
Cedro & 6,42 \\
Convivencia & 5,78 \\
Curso Tutores & 8,68 \\
Del Papel a la Nube & 6,64 \\
Diseño Aprendizajes & \\
\hline
\end{tabular}




\begin{tabular}{lr} 
Educa Madrid & 8,89 \\
Espacios Web & 8,54 \\
Evaluación & 8,55 \\
Evaluar para Aprender & 8,15 \\
Flipped Classroom & 8,63 \\
Función Tutorial & 8,21 \\
Funcionario Prácticas & 8,23 \\
Instituto Nacional Estadística & 7,44 \\
Moodle & 8,33 \\
Paisajes Aprendizaje & 8,71 \\
Propiedad Intelectual & 8,1 \\
Tecnol. Program.y Robótica & 5,23 \\
Transformación Digital & 6,48 \\
Web Aula & 8,87 \\
Web Centro & 8,87 \\
Web Docente & 8,56 \\
Total & 7,96 \\
\hline
\end{tabular}

Estudiando este valor respecto a la temática de los cursos, vemos que los cursos con temática tecnológica son los más valorados, y además los que menos dispersión de opinión tienen.

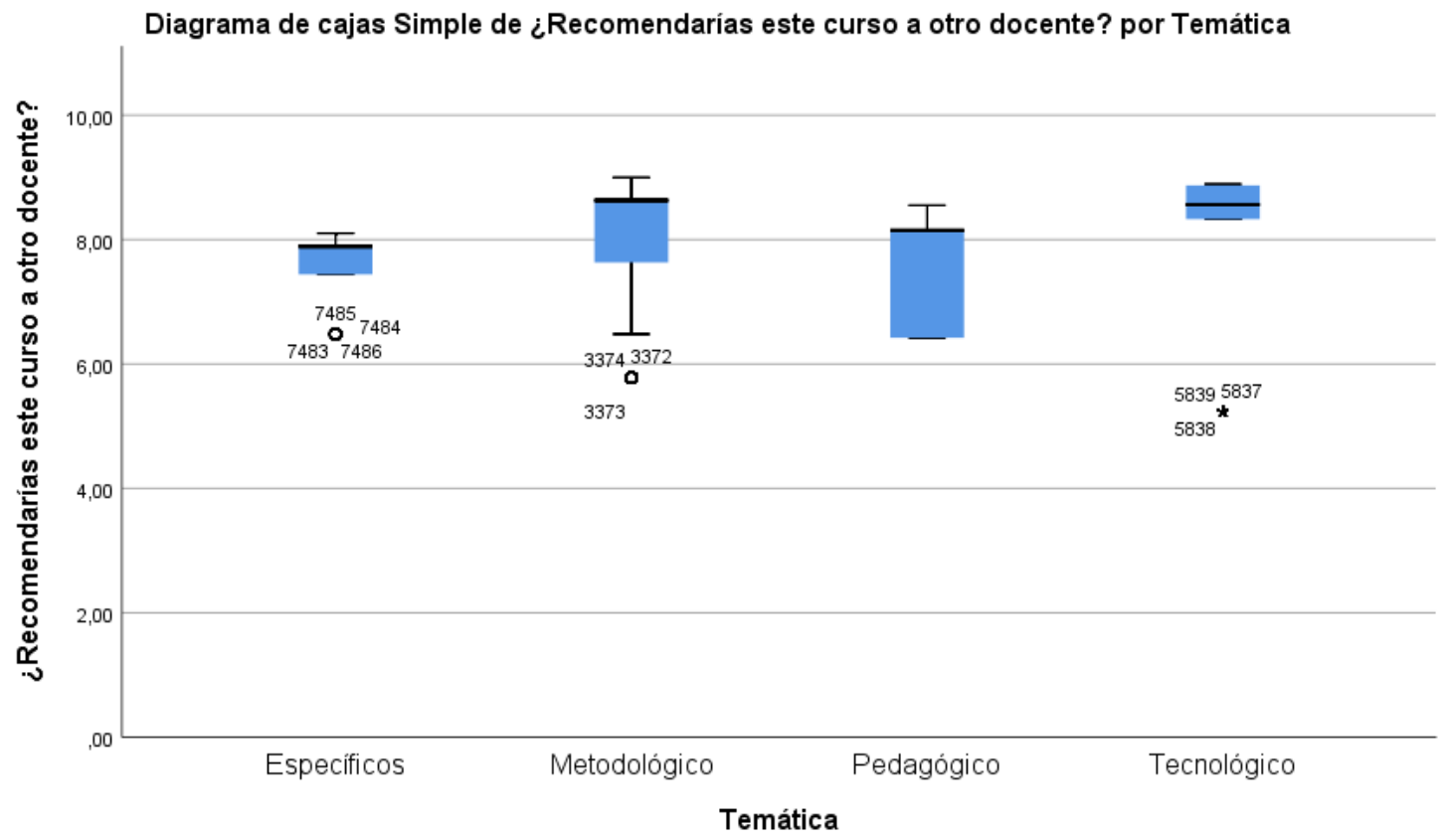

Figura 4. Diagrama por temáticas. “:Recomendarías este curso a otro participante?”

\section{Discusión}

Para que el profesor incorpore las tecnologías de la información y la comunicación, es fundamental que él mismo experimente una variedad de modalidades e iniciativas en su 
formación. Las capacidades y competencias que el docente tendrá que movilizar para enseñar en la nueva sociedad no se adquieren en contextos formativos que no las promuevan. Se requieren pues nuevos contextos de formación en un entorno digital (Menéndez-Ferreira, Fuente, Gómez, y Camacho 2017). García-Valcárcel, et al. 2015; Vaillant, 2014). Esto justifica claramente la necesidad de un plan de formación en línea de docentes, en el que las herramientas TIC han de servir para motivar, estimular e involucrar en el proceso de enseñanza, facilitando la comprensión y el aprendizaje significativo (Esteve y Gisbert, 2011). Esto se alinea con los datos que señala nuestro estudio sobre el plan de formación, que muestra que los docentes se guían de la administración educativa para orientar su formación, ya que casi la mitad de ellos se guía y orienta a través de la web oficial de la Consejería.

Este plan de formación en línea está diseñado para mejorar la calidad docente y por ello es importante conocer la percepción que tienen los participantes en la formación, no sólo de manera global, sino diferenciando los distintos elementos del curso. Los contenidos, las actividades y los trabajos que se plantean en los cursos son los mejor valorados por los participantes, coincidiendo en importancia con el estudio de Rivero y Mur (2015). Este estudio en cambio destaca la importancia de la metodología, además de los contenidos, como base para planteamientos innovadores en formación.

La alta valoración de los contenidos, otorga una importancia a dichos elementos digitales que se ve reforzado en otras invesitigaciones que muestran que los profesores siguen valiéndose mayoritariamente de las plataformas como meros repositorios de objetos de aprendizaje o de recolección de información, es decir, como simples transmisores de información (Brooks y Pomerantz, 2017).

El formato del curso es en cambio el aspecto con peores resultados en la valoración de los docentes, lo cual resulta contradictorio con la importancia que le otorgan estudios como el de Area, Borrás, y San Nicolás (2014), que además remarcan su valor porque permite al aprendiz tener autonomía y flexibilidad en la cumplimentación de las tareas similares a las que se encontrará en su futura actividad como maestro utilizando las TIC. Esta segunda parte del estudio que perfila su utilidad en el aula, sí se ve refrendada en nuestros datos, ya que utilizar actividades similares a las actividades de aula es el elemento mejor valorado.

Los docentes también le dan la máxima importancia a las actividades planteadas. Nos encontramos con resultados similares a los obtenidos por Area (2010), el cual indica además que la configuración de entornos de aprendizaje online requieren que el docente planifique el aula virtual a partir de actividades que los estudiantes deben cumplimentar y no en torno a los núcleos temáticos.

La participación de los docentes en procesos de formación en TIC tiene importante incidencia en las prácticas de enseñanza, pues cerca del 95\% de los profesores formados ha puesto en práctica lo aprendido o está en vías de hacerlo. Estos datos tan altos pueden tener su justificación en estudios como el de Guerra, González y García (2010) que comparte la importancia de que la formación vaya acompañada de una aplicación al aula, una vez que el profesorado se enfrenta a la preparación de su asignatura o proyectos de investigación con recursos TIC. En cambio difiere de otros estudios como el de Coronado, Cantú y Rodríguez (2014) que indica índices menores de aplicación. La diferencia podría no ser tal si consideramos como "no transferencia" a los docentes que dicen estar a la espera de poner en práctica lo aprendido.

Respecto a las temáticas formativas, los cursos técnicos no son los más solicitados ni los más impartidos, a pesar de que Fernández-Cruz y Fernández-Díaz (2016) indican que una de las premisas para la incorporación de las TIC al mundo educativo es poseer conocimientos 
técnicos de las herramientas digitales. Los cursos con temática metodológica y didáctica son claramente los cursos más valorados y más solicitados por los docentes, y los que más transferencia tienen al aula, con más de un $40 \%$ y casi 15 puntos por encima del resto. Esto sí está alineado con otros estudios que hablan de que la capacitación en la formación virtual de los docentes se debe centrar en el componente didáctico, el más significativo para que el docente incorpore las herramientas digitales en su práctica educativa, y no tanto en el componente instrumental. (Del Prete y Almenara, 2019; Cabero-Almenara, Gallego-Pérez, Puentes-Puente y Jiménez-Rosa, 2018; Cabero y Marín, 2014)

La formación del profesorado debe ir por tanto orientada tanto a la formación instrumental, en cuanto al uso y manejo de las herramientas tecnológicas como a la capacitación pedagógica necesaria para emplear e integrar estos recursos en los procesos de enseñanzaaprendizaje. (Del Moral y Villalustre, 2010). Esto coincide con Ríos, Gómez y Rojas (2018) en un estudio realizado en Chile, en el que cerca del 35\% del profesorado se inclina por tener una alfabetización digital alta, afirmando tener un mayor dominio de la dimensión tecnológica y la informacional y un menor dominio en la dimensión pedagógica (a pesar de que el 93\% del profesorado de la muestra tiene capacitación pedagógica).

Como conclusión final podríamos decir que para abordar con garantías el actual marco educativo, los docentes valoran fundamental tener una buena formación tecnopedagógica que les permita tomar el control en el uso de recursos tecnológicos en sus aulas; y paralelamente una formación de índole técnica sobre el uso de diversas herramientas digitales que les dé seguridad en la puesta en práctica de las sesiones. Todo ello al servicio de una experiencia pedagógica innovadora.

\section{Referencias}

Alharbi, S., \& Drew, S. (2014). Using the Technology Acceptance Model in Understanding Academics' Behavioural Intention to Use Learning Management Systems. International Journal of Advanced Computer Science and Applications (IJACSA), 5(1). https://doi.org/10.14569/IJACSA.2014.050120

Area, M. (2010). "Del HTml a la web 2.0: autobiografía de una década de docencia universitaria con Tic". En R. Roig Vila y M. Fiorucci, Claves para la investigación en innovación y calidad educativas. La integración de las Tecnologías de la Información y la Comunicación en la interculturalidad en las aulas (pp. 10-21).

Area, M., Borrás, J. F. y San Nicolás, B. (2014). La formación del maestro 2.0: el aprendizaje por tareas en entornos b-learning. Revista Interuniversitaria de Formación del Profesorado, 79 (28.1), 51-66

Blau, I., \& Shamir-Inbal, T. (2016). Digital competences and long-term ICT integration in school culture: The perspective of elementary school leaders. Education and Information Technologies, 22. https://doi.org/10.1007/s10639-015-9456-7

Brooks, D. C. \& Pomerantz, J. (2017). ECAR Study of Undergraduate Students and Information Technology. Louisville, CO. Educause.

Cabero, J. y Marín, V. (2014). Miradas sobre la formación del profesorado en TIC. Revista Venezolana de Información, Tecnología y Conocimiento, 11 (2), págs. 11-24

Cabero-Almenara, J., Estrada-Vidal, L. Gutiérrez-Castillo, J.J. (2017). Diseño y validación de un instrumento de evaluación de la competencia digital del estudiante universitario. Revista Espacios, 38(10), 16. doi: https://doi.org/10.11144/Javeriana.m8-17.ctap 
Cabero-Almenara, J., Gallego-Pérez, O., Puentes Puente, Á., y Jiménez Rosa, T. (2018). La "Aceptación de la Tecnología de la Formación Virtual" y su relación con la capacitación docente en docencia virtual. EDMETIC, Revista de Educación Mediática y TIC, 7(1), 225-241, https://doi.org/10.21071/edmetic.v7i1.10028

Castells, M. (2003) La era de la información. Belen: Alianza Editorial.

Coll, C; Onrubia, J.; Mauri, T. (2007). Tecnología y prácticas pedagógicas: las TIC comoinstrumentos de mediación de la actividad conjunta de profesores y estudiantes. Anuario de Psicología vol. 38 (3), pp. 377-400.

Coronado, E., Cantú, M., y Rodríguez, C. (2014). Diagnóstico universitario sobre el uso de la TIC en el proceso de enseñanza-aprendizaje bajo la modalidad educativa presencial en Santo Domingo. Revista Edutec, 50, pp. 70-81.

Del Moral, E. y Villalustre, L. (2010). La formación del profesor 2.0. Desarrollo de competencias tecnológicas para la Escuela2.o. Revista Miscelánea de Investigación, 23, pp. 59-70.

Del Prete, A.; Cabero-Almenara J. y Halal, C. (2018). Motivos inhibidores del uso del Moodle en docentes de educación superior. Campus Virtuales. Revista Científica Iberoamericana de Tecnología Educativa, 7(2), pp. 69-80.

Esteve, F.M. y Gisbert, M.(2011). El nuevo paradigma de aprendizaje y las nuevas tecnologías.Revista de Docencia Universitaria. REDU. Monográfico: El espacio europeo de educación superior. Hacia donde va la Universidad Europea?. 9 (3), 55-73. Recuperado de http://redaberta.usc.es/redu

Fernández-Cruz, F.J, Fernández-Díaz, M.J. (2016) Los docentes de la Generación Z y sus competencias digitales. Revista Comunicar n¹6, pp. 97-105. http://dx.doi.org/10.3916/C46-2016-10

Fullan, M. G., \& Huberman, M. (1992). Successful School Improvement: The Implementation Perspective and Beyond. Buckingham England; Philadelphia: Open University Press.

García-Valcárcel, A., Basilotta, V., Cabezas-González, M., Casillas, S., González, L., Hernández, A., \& Mena, J. J. (2014). La formación del profesorado universitario en Tecnologías de la Información y la Comunicación en la Universidad de Salamanca. RELATEC, Revista Latinoamericana de Tecnología Educativa, 14(1), 75-88.

Guerra, S., González, N. y García, R. (2010). Utilización de las TIC por el profesorado universitario como recurso didáctico. Revista Comunicar, 35, 141-148.

Hargreaves, A. (2005). Profesorado, cultura y postmodernidad: Cambian los tiempos, cambia el profesorado (Edición: 1). España: Ediciones Morata, S.L.

How the world's best-performing school systems come out on top | McKinsey. (s. f.). Recuperado 6 de marzo de 2020, de https://www.mckinsey.com/industries/socialsector/our-insights/how-the-worlds-best-performing-school-systems-come-out-ontop

Kang, M., \& Shin, W. S. (2015). An Empirical Investigation of Student Acceptance of Synchronous E-Learning in an Online University. Journal of Educational Computing Research, 52. https://doi.org/10.1177/0735633115571921

Menéndez-Ferreira, R., Fuente, A., Gómez, M. y Camacho, D. (2017). Improving Sociocultural Outcomes for Students in the Higher Education through Participation on Virtual 
Mobility: The UbiCamp Experience. International Journal of Engineering Education vol, 33, pp. 2050-2060.

Park, J. Y. (2014). Course evaluation: reconfigurations for learning with learning management systems. Higher Education Research \& Development, 33(5), 992-1006. https://doi.org/10.1080/07294360.2014.890564

Park, S. Y., Nam, M.-W., \& Cha, S.-B. (2012). University students' behavioral intention to use mobile learning: Evaluating the technology acceptance model. British Journal of Educational Technology, 43(4), 592-605. https://doi.org/10.1111/j.14678535.2011.01229.x

Parsons, A. (2017). Accessibility and use of VLEs by students in further education. Research in Post-Compulsory Education, 22(2), 271-288. https://doi.org/10.1080/13596748.2017.1314684

Persico, D., Manca, S., \& Pozzi, F. (2014). Adapting the Technology Acceptance Model to evaluate the innovative potential of e-learning systems. Computers in Human Behavior, 30, 614-622. https://doi.org/10.1016/j.chb.2013.07.045

Prete, A. D., \& Almenara, J. C. (2019). Las plataformas de formación virtual: algunas variables que determinan su utilización. Apertura: Revista de Innovación Educativa, 11(2), 138153. Recuperado de https://dialnet.unirioja.es/servlet/articulo?codigo=7099625

Ramos-Pardo, F. J.; Sánchez, P. y Sánchez, J. (2012). Usos pedagógicos de Moodle en la docencia universitaria desde la perspectiva de los estudiantes. Revista Iberoamericana de Educación, 60, pp. 15-38.

Ríos, J.M., Gómez, E.R., \& Rojas, M.P. (2018).Valoración de competencias TIC del profesorado universitario: un caso en Chile. Píxel-Bit. Revista de Medios y Educación, 52, pp. 55-65. http:// dx.doi.org/10.12795/

Redecker, C y Punie, Y. (2017). Digital Competence of Educators DigCompEdu. Luxembourg: Publications Office of the European Union.

Rienties, B., Giesbers, B., Lygo-Baker, S., Ma, H. W. S., \& Rees, R. (2016). Why some teachers easily learn to use a new virtual learning environment: a technology acceptance perspective. Interactive Learning Environments, 24(3), 539-552. https://doi.org/10.1080/10494820.2014.881394

Ríos Ariza, J. M., Gómez Barajas, E. R., \& Rojas Polanco, M. P. (2018). Valoración de competencias TIC del profesorado universitario: un caso en Chile. https://doi.org/10.12795/pixelbit.2018.i52.04

Rivero, P., \& Mur, L. (2015). Aprender ciencias sociales en la web 2.0 / Learning Social Sciences with web 2.o. Íber. Didáctica de las Ciencias Sociales, Geografía e Historia, 80, 30-37.

Rodríguez, A.; Raso, F. y Ruiz-Palmero, J. (2019). Competencia digi- tal, educación superior y formación del profesorado: un estudio de meta-análisis en la Web of Science. PixelBit. Revista de Medios y Educación, 54, pp. 65-81.

Sinclair, J., \& Aho, A.-M. (2018). Experts on super innovators: understanding staff adoption of learning management systems. Higher Education Research \& Development, 37(1), 158-172. https://doi.org/10.1080/07294360.2017.1342609

Solís de Ovando, J., y Jara, V. (2019). Competencia digital de docentes de Ciencias de la Salud de una universidad chilena. Pixel-Bit, Revista de Medios y Educación, 56, 193-211. doi: https://doi.org/10.12795/pixelbit.2019.i56.10 
Tarhini, A., Hone, K., \& Liu, X. (2014). Measuring the Moderating Effect of Gender and Age on E-Learning Acceptance in England: A Structural Equation Modeling Approach for An Extended Technology Acceptance Model: Journal of Educational Computing Research. https://doi.org/10.2190/EC.51.2.b

Unión Europea (2018). Recomendación del Consejo de 22 de mayo de 2018 relativa a las competencias clave para el aprendizaje permanente. Diario Oficial de la Unión Europea. Comisión Europea.

Vaillant, D. (2014). FORMACIÓN DE PROFESORES EN ESCENARIOS TIC. Revista e-Curriculum, 12(2), 1128-1142. Recuperado de https://revistas.pucsp.br/curriculum/article/view/20850

Valencia, A., Benjumea, M. y Rodríguez, V. (2014). Intención de uso del e- learning en el programa de administración tecnológica desde la perspectiva del modelo de aceptación tecnológica. Revista Electrónica Educare, 18(2), 247-264. http://dx.doi.org/10.15359/ree.18-2.13 